\title{
Monte Carlo PENRADIO software for dose calculation in medical imaging
}

\author{
Camille Adrien, Mercedes Lòpez Noriega, Guillaume Bonniaud, Jean-Marc Bordy, Cindy Le Loirec, \\ Bénédicte Poumarede
}

\begin{abstract}
The increase on the collective radiation dose due to the large number of medical imaging exams has led the medical physics community to deeply consider the amount of dose delivered to patients as well as its associated risks in these exams. For this purpose we have developed a Monte Carlo tool, PENRADIO, based on a modified version of the 2006 release of the PENELOPE code, to obtain an accurate individualized radiation dose in conventional and interventional radiography and in computed tomography (CT). This tool has been validated showing excellent agreement between the measured and simulated organ doses in the case of a hip conventional radiography and a coronography. We expect the same accuracy in further results for other localizations and in CT examinations.
\end{abstract}

\section{INTRODUCTION}

$\mathbf{T}$ HE significant rise of medical imaging exams in the past few years has led to an increase in the collective dose due to irradiation [1], [2], [3]. The medical physics community agreed that this increase must be accompanied by a strong understanding of the radiation dose and its associated risks [4], [5], [6], [7]. A controversial article announced that around $1.5 \%$ of all cancers in the United States may be attributable to the radiation from computed tomography (CT) examinations [5]. Even if this was an overestimation, it points out the necessity of convenient tools to evaluate the associated dose of medical radiation. Commonly, non-individualized indices are used to estimate the radiation dose (CT dose index (CTDI), dose-length product (DLP), dose-area product (DAP), entry dose, etc ...). The effective dose was first introduced as a measurement of dose for radioprotection, reflecting the amount of radiation detriment. However it is mainly used to directly compare various radiation types and exposures but does not reflect the actual absorbed dose. Several applications have been developed and provide the common dose index (CTDI, DLP, dose-surface product), organ and tissue absorbed and/or effective dose.

For conventional radiography (CR): PCXMC estimates the organ dose, the effective dose and its associated risk in $\mathrm{CR}$ for 29 organs and various types of numerical phantoms[8].

C. Adrien is with the CEA LIST, Gif-sur-Yvette, F-91191 France (telephone: 0033169082311, e-mail: camille.adrien@cea.fr).

M. Lòpez Noriega was with the CEA LIST, Gif-sur-Yvette, F-91191 France (e-mail: mercedesln@gmail.com)

G. Bonniaud is with Centre de Medecine Nucleaire du Morbihan, Centre Hospitalier Bretagne Atlantique et Centre d Oncologie Saint-Yves, Vannes, F-56000 France (e-mail: guillaume.bonniaud@esprimed.net).

JM. Bordy is with the CEA LIST, Gif-sur-Yvette, F-91191 France (telephone: 0033169084189, e-mail: Jean-Marc.BORDY@cea.fr).

C. Le Loirec is with the CEA LIST, Gif-sur-Yvette, F-91191 France (telephone: 0033169087850, e-mail: cindy.le-loirec@cea.fr).

B. Poumarede is with the CEA LIST, Gif-sur-Yvette, F-91191 France (telephone: 0033169084990, e-mail: Benedicte.POUMAREDE@cea.fr).
XDOSE and CHILDDOSE are based on the same principle. The CALDose_ $X$ calculates the common dose index in radiology and estimates the organ doses[9].

For CT examinations: ImPACT, Eff-Dose [10] and OrgDose[11] give CTDI and DLP for several CT. ImpactMC provides a dose delivered estimation after examination[12]. Several research teams use Monte Carlo (MC) simulations for CT examinations [13], [14], [15], [16]. For instance, the Duke University Medical Center has developed a tool for estimating organ absorbed dose and effective dose based on the PENELOPE code and using adaptive anthropomorphic numerical phantoms [16].

Most of already existing tools do not take the person biological specificities into account and thus, only provide common dose index and effective dose rather than an actual dose delivered. Therefore our goal is not only to gather in a single software all these proposed features but also to replace common dose index with actual organ absorbed doses. For that purpose we decided to include in a new MC tool, PENRADIO, the possibility of introducing individual specificities in order to obtain personalized results. The present MC program, dedicated to medical $\mathrm{x}$-ray imaging procedures, is based on a modified version of the 2006 release of the PENELOPE code[17]. It allows the calculation of an accurate individualized radiation dose in voxelized numerical phantoms. We start by presenting the tool and the validation methodology for CR and interventional radiography (IR) as well as preliminary results. Then, CT tool developments are described.

\section{METHOD}

\section{A. The PENRADIO Software}

The PENRADIO software (Figure 1) is based on PENSSART, a software initially developed for safety in radiotherapy [18]. The program uses PENELOPE code 2006 release allowing for particle transport in voxelized geometries. The PENSSART software is divided into three modules:

- The dose calculation module is the core of the program. It was designed to perform MC dose calculations within voxelized geometries. To add this new functionality to PENELOPE a specific main program was developed in $\mathrm{C}++$. The physics initially developed in PENELOPE remain unchanged and a ray-tracing algorithm was added for efficient navigation in the voxelized geometry. Results are provided in the form of two output matrices storing the dose absorbed in the medium and the statistical uncertainty for each voxel. The dose absorbed in the medium can be expressed in $\mathrm{eV} / \mathrm{g} /$ shower $D_{M C, e V / g / s h w}$ or in Gray $D_{a b s, G y}$. Normalization factors [19] are usually computed for the conversion from simulation results to absolute dose 
values. We have chosen another method to determine the absolute dose values. When a phase space file (PSF) is used as an input, we can use:

$$
D_{a b s, G y}=1.6 \cdot 10^{-16} \cdot N_{e l e c} \cdot D_{M C, e V / g / s h w}
$$

with $N_{\text {elec }}$ the number of electrons corresponding to the mAs used for the image acquisition, thus:

$$
N_{\text {elec }}=A \cdot s / 1.6 \cdot 10^{-19}
$$

When an energy spectrum is used, the result is given as the absorbed dose per initial photons. In addition to the number of initial electrons provided by the examination acquisition protocol, the tube yield $y_{T X}$, ratio of photons leaving the tube to initial electrons impinging the anode, has to be determined. The absolute absorbed dose can hence be obtained as follows:

$$
\begin{gathered}
D_{a b s, G y}=1.6 \cdot 10^{-16} \cdot N_{p h} \cdot D_{M C, e V / g / s h w} \\
D_{a b s, G y}=1.6 \cdot 10^{-16} \cdot y_{T X} \cdot N_{\text {elec }} \cdot D_{M C, e V / g / s h w}
\end{gathered}
$$

- The patient module allows the implementation of complex geometries. The user can convert quadratic geometries normally used in PENELOPE into voxelized geometries by using a routine provided in PENCT [20] (such geometries will be called pengeom 2 ct phantoms ${ }^{1}$ ). A conversion process to transform patient $\mathrm{CT}$ images into data usable by the MC dose computation module has also been implemented[18].

- The radiation source module allows the simulation of different kinds of radiation source going from simple sources such as monoenergetic beams to more complex sources like PSF resulting from a complete MC modeling of the $\mathrm{X}$-ray tube. To generate such PSF, the module uses the PENELOPE code with a new version of the main program PENMAIN in which the selective bremsstrahlung splitting was implemented for increasing simulation efficiency.

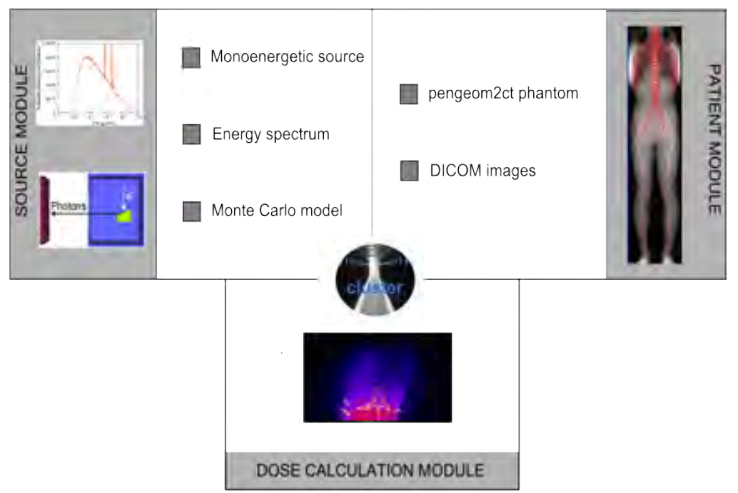

Fig. 1. PENRADIO units and connections for dose calculation.

\section{B. Dose calculation in $C R$ and $I R$}

In $\mathrm{CR}$ and IR, the information provided in the technical notes of the manufacturer is sufficient to perform a complete MC model of an X-ray tube.
1) Monte Carlo simulations: A CR tube (Philips DiDi TH2 CS4) and an IR tube (Philips MRC 2000508 ROT-GS 1003) were simulated with PENELOPE according to the information provided in the technical notes. For the purpose of this study, several PSF corresponding to the maximal field size at the tube output were generated:

- two PSF for $77 \mathrm{kVp}$ and $102 \mathrm{kVp}$ of the CR tube;

- five PSF: four from 70 to $85 \mathrm{kVp}$ in steps of $5 \mathrm{kVp}$ and one for $95 \mathrm{kVp}$ for the IR tube.

Simulation with PENELOPE is controlled by different parameters: the absorption energies $E_{a b s}$ for electrons, photons and positrons, $C_{1}$ and $C_{2}$ determining the mean free path between hard elastic events and the maximum average fractional energy loss in a single step, $W_{C C}$ and $W_{C R}$ which are cutoff energies for hard inelastic interactions and hard bremsstrahlung emissions respectively. The simulation parameters are defined as a function of $E_{\max }$, the maximum energy of the electron such as $E_{a b s, \text { electron }}=E_{\text {abs,positron }}=E_{\max } / 100$, $E_{\text {abs }, \text { photon }}=E_{\max } / 1000, W_{C C}=E_{\max } / 100, W_{C R}=$ $E_{\text {max }} / 1000, C_{1}=C_{2}=0.2$ for anode material and 0.1 for others.

2) Validation of the MC models: The main parameters of the MC model have been adjusted and validated using half value layers (HVL). The UNFORS detector has been used to measure the HVL of each simulated beam. The measurements have been compared with MC simulations performed with several aluminium filtrations. For each filtration the air Kinetic Energy Released per unit MAss (KERMA) has been calculated. Plotting air KERMA variations as a function of the aluminium filtration allows HVL determination.

3) Simulation of organ doses: Physical anthropomorphic phantoms (CIRS ATOM dosimetry phantoms) and their DICOM images have been used to compare measured and simulated radiation doses in the case of a hip CR (Table I) and in the case of a cardiac IR (Table II). Measurements were performed with Optically Stimulated Luminescence (OSL) dosimeters inserted in the phantoms [21] while the PENRADIO software was used to determine the dose in some organs of interest [22], [23]. Information about the calibration and the correction of the OSL readings is reported in the Appendix.

\begin{tabular}{cc}
\hline HighVoltage $(\mathrm{kVp})$ & Charge (mAs) \\
\hline 77 & 25.2 \\
102 & 6.91 \\
\hline \multicolumn{3}{c}{ TABLE I } \\
CR ACQUISITION TUBE PARAMETERS.
\end{tabular}

\section{Tool adaptation for CT exams}

The full MC modeling of the GE Lightspeed VCT 64 CT tube is impossible with the information provided in the technical notes of the manufacturer, especially because of the particular shape of the bowtie filters. In order to overcome this problem, we have implemented the method proposed by Turner et al [24]. Their study presents a method for generating x-ray source models based on experimental data. The so-called "Turner method" enables us to get equivalent spectra and bowtie filters. 


\begin{tabular}{ccc}
\hline HighVoltage $(\mathrm{kVp})$ & Charge (mAs) & Acquisition Mode \\
\hline 73 & 154 & Graphy \\
70 & 100 & Graphy \\
73 & 128 & Graphy \\
73 & 64 & Graphy \\
74 & 89 & Graphy \\
93 & 13 & Scopy \\
72 & 101 & Graphy \\
77 & 165 & Graphy \\
74 & 119 & Graphy \\
\hline
\end{tabular}

TABLE II

IR ACQUISITION TUBE PARAMETERS.

1) Experimental determination of the $X$-ray tube spectrum: The equivalent inherent filtration of the tube has been determined using a program based on Turner work [24]. Three inputs are mandatory to run the developed program:

a. the first and second half value layers $\left(\mathrm{HVL}_{1}\right.$ and $\left.\mathrm{HVL}_{2}\right)$;

b. an initial tungsten anode $\mathrm{X}$-ray energy spectrum;

c. an arbitrarily chosen material used for filtration.

The following steps, reported in the flowchart of Figure 2, are used to get an equivalent spectrum: [1] The input energy spectrum is filtered assuming exponential attenuation by an initial thin sheet of aluminium (IF) providing a candidate spectrum and its associated KERMA $\left(K_{0}\right)$. [2] Then the candidate spectrum is repetitively filtered by increasing the aluminium sheet thickness (AF) and its associated KERMA ( $\left.K_{1}\right)$ is calculated until $K_{0}=2 K_{1}$. The simulated $\mathrm{HVL}_{1}$ is set to the global additional sheet thickness used to verify this condition. [3] The second step is repeated to evaluate the simulated $\mathrm{HVL}_{2}$ which fulfils the condition $K_{0}=4 K_{2}$. [4] Then comparison between experimental and computed data has to be made: either only $\mathrm{HVL}_{1}$ values are compared (Method A) or the sum of both $\mathrm{HVL}_{1}$ and $\mathrm{HVL}_{2}$ values (Method B). If experimental and simulated data are equivalent, the equivalent spectrum is found. Otherwise, a new thicker initial sheet of aluminium is tested (step 1).

To run the Turner method we chose to use two different spectra to initialize the process, one softly filtered obtained with the SpekCalc tool[25], the other unfiltered obtained using MC simulation in order to compare the influence of the primary spectrum in the simulation process. The MC simulation got using PENELOPE code with a geometry based on the technical notes provides both the MC spectrum and the PSF of the CT head. The PSF matches a $4 \mathrm{~cm}$ x $50 \mathrm{~cm}$ field size at a distance of $54.1 \mathrm{~cm}$ from the source for a $120 \mathrm{kVp}$ voltage. The simulation parameters are defined as $E_{a b s, \text { electron }}=E_{a b s, \text { positron }}=1000$ $\mathrm{eV}, E_{\text {abs,photon }}=100 \mathrm{eV}, W_{C C}=W_{C R}=100 \mathrm{eV}$ and $C_{1}=C_{2}=0.2$.

Both SpekCalc and PENELOPE equivalent spectra have been used in the PENRADIO software to determine the simulated air KERMA $\left(K_{a i r, s i m}\right)$ at the CT isocenter. In a static mode, simulated values and the experimental KERMA values, measured with a NE-2571 ionization chamber have been compared to determine the best suited method to model the X-ray tube.

2) Experimental determination of two bowtie filters: The determination of an equivalent bowtie filter consists in obtaining

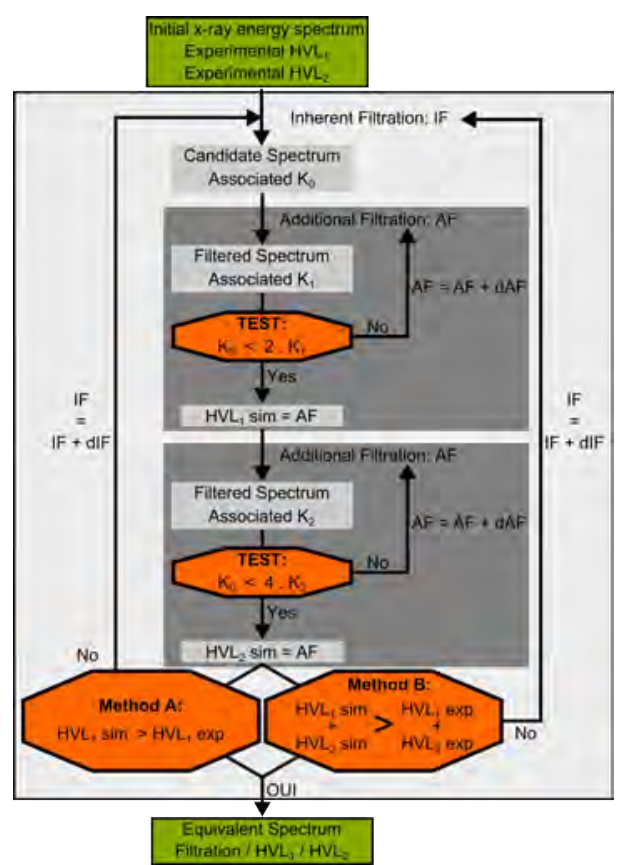

Fig. 2. Flowchart for the experimental determination of the CT model.

the aluminium thickness as a function of $\theta$ which attenuates the equivalent spectrum in the same manner that the actual bowtie filter. The equivalent bowtie filter has also been determined using a program based on Turner work [24]. Two inputs are necessary to run the program: the equivalent energy spectrum previously computed and the bowtie profile measurements.

The following steps are repeated for each angle: [1] the ratio $(\mathrm{R})$ of the measured point at $\theta_{i}$ to the measured central points is computed. [2] The KERMA $\left(\mathrm{K}_{0}\right)$ associated to the equivalent spectrum is calculated. [3] The equivalent spectrum is repetitively filtered by increasing the aluminium sheet thickness $\left(\mathrm{e}_{A l}\right)$ and its associated KERMA $\left(K_{1}\right)$ is calculated until $K_{0}=R K_{1}$. [4] The aluminium thickness for $\theta_{i}$ is set to $\mathrm{e}_{A l}$. Both head and body bowtie filter shapes have been determined and designed with PENGEOM, the PENELOPE package that handles the geometry.

3) Implementation of the rotation in PENRADIO: To model the effect of x-ray tube motion during an axial or helical scan, the position and direction of each particle stored in the input PSF were transformed before the particle was released for transport in the patient or phantom. Rotational and translational transformations were performed according to the following equations [16]:

$$
\begin{gathered}
\beta=\alpha \cdot R A N D \\
d=\beta / 2 \pi \cdot s+z_{0}
\end{gathered}
$$

with $\alpha$ the total gantry angle rotation during the scan, $R A N D$ a random value between 0 and $1, s$ the table increment per gantry rotation and $z_{0}$ the start location of the scan. For single axial scans, $\alpha$ and $s$ equal $2 \pi$ and 0 , respectively. 


\section{RESULTS}

Results of dose calculations with the PENRADIO software in CR, IR and CT as well as their validation are presented.

\section{A. Dose calculation in $C R$ and $I R$}

1) Validation of the model: The results obtained for the validation of the CR and IR tubes are reported in Table III. The difference between the measurements and the simulations varies between 0.2 and $5.5 \%$.

\begin{tabular}{lcccc}
\hline & \multicolumn{2}{c}{ CR } & \multicolumn{2}{c}{ IR } \\
\hline & & & graphy & scopy \\
\hline Potential & $77 \mathrm{kV}$ & $102 \mathrm{kV}$ & $74 \mathrm{kV}$ & $85 \mathrm{kV}$ \\
\hline Exp. data (mm Al) & 3.70 & 4.85 & 4.68 & 9.37 \\
Sim. data (mm Al) & 3.65 & 5.00 & 4.95 & 9.35 \\
Deviation (\%) & 1.35 & 3.10 & 5.50 & 0.20 \\
\hline
\end{tabular}

TABLE III

Measured And Simulated HVL USED to VAlidate THE CR AND IR TUBES.

2) Simulation of organ doses: Measured and simulated doses in the case of a hip conventional radiography and in the case of a fluorography during a cardiac interventional radiology procedure are presented in Table IV, Figure 3 and Figure 4. For IR examination MC dose error and the deviation between simulated and measured errors are compatible. Nevertheless, for CR exam, MC dose error are lower than the deviation between simulated and measured doses, this could be explained by a low MC statistic. However, in a context of radioprotection, errors about $20 \%$ are tolerated for medical imaging examinations.

\begin{tabular}{cccc}
\hline Localization & $\begin{array}{c}\text { Measured } \\
\text { Dose (mGy) }\end{array}$ & $\begin{array}{c}\text { Simulated } \\
\text { Dose (mGy) }\end{array}$ & $\begin{array}{c}\text { Deviation } \\
(\%)\end{array}$ \\
\hline Hip [CR] & 0.233 & $0.29 \pm 0.03$ & 23.6 \\
Lung [IR] & 0.584 & $0.56 \pm 0.05$ & 3.5 \\
\hline
\end{tabular}

TABLE IV

MEASURED AND SIMULATED DOSE FOR A HIP CONVENTIONAL RADIOGRAPHY AND A FLUOROGRAPHY IN A CARDIAC INTERVENTIONAL RADIOGRAPHY.

\section{B. Tool adaptation for CT exams}

1) Equivalent Spectra: Both SpekCalc and MC initial and equivalent spectra are shown in Figure 5. Values of inherent filtration, first and second HVL are reported in Table V. Values highly depend on the test performed but not on the spectrum type:

- When only the first HVL is used to determine the inherent filtration, the algorithm converges on the experimental value for the first HVL and a deviation of $7.7 \%$ is obtained for the second HVL estimation. These results are identical for both SpekCalc and MC spectra.

- When both HVL are used together for inherent filtration determination, a deviation of $6.6 \%$ and $5.0 \%$ can be noticed for the first and the second HVL, respectively.

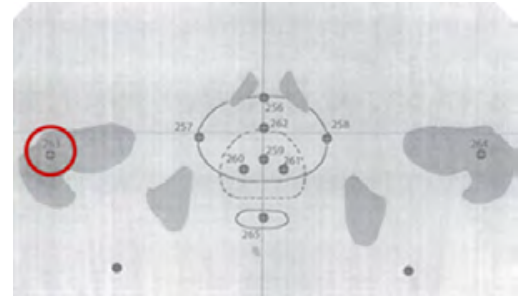

(a) OSL position in phantom

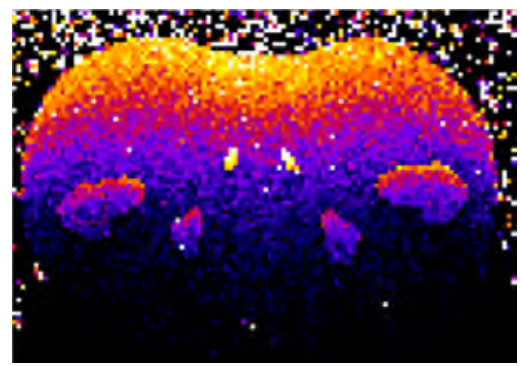

(b) Dose map

Fig. 3. Male patient in a hip conventional radiography examination at $77 \mathrm{kV}$.

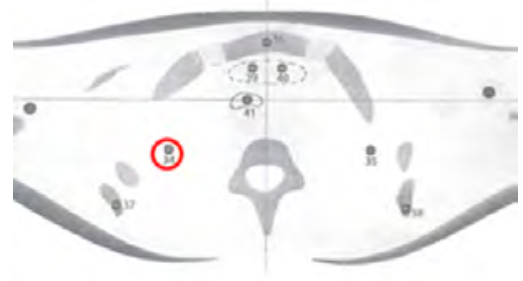

(a) OSL position in phantom

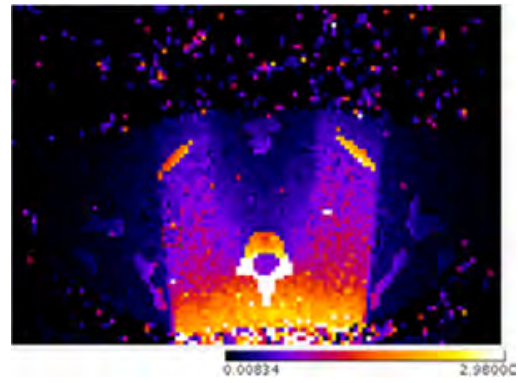

(b) Dose map (mGy)

Fig. 4. Female patient in cardiac interventional radiography examination at $68 \mathrm{kV}$.

Both HVL values are comparable to each other for both SpekCalc and MC spectra.

Due to the common use of maximal potential and first HVL to qualify a x-ray tube and a greater global error for the method including both HVL, in the following we chose to use equivalent spectra obtained with the algorithm based on a comparison only of the first HVLs.

Measured air KERMA has been compared to simulated values based on both SpekCalc and MC equivalent spectra obtained using Method A (Table VI). Simulated values are given in $\mathrm{eV} / \mathrm{g} /$ photon and are converted into Gray using Equation 3. $y_{T X}$ was set to $3.14710^{-5}$ for the equivalent filtration of 1.94 


\begin{tabular}{lccccc}
\hline & Exp. & \multicolumn{2}{c}{ Spekcalc } & \multicolumn{2}{c}{ Monte Carlo } \\
\hline Method & & A & B & A & B \\
\hline Filtration $(\mathrm{mm} \mathrm{Al})$ & & 2.10 & 1.67 & 1.94 & 1.51 \\
\hline First HVL $(\mathrm{mm} \mathrm{Al})$ & 6.08 & 6.08 & 5.68 & 6.08 & 5.69 \\
Second HVL (mm Al) & 8.23 & 8.86 & 8.64 & 8.87 & 8.63 \\
\hline
\end{tabular}

TABLE V

RESULTS OBTAINED FOR THE INHERENT FILTRATION.

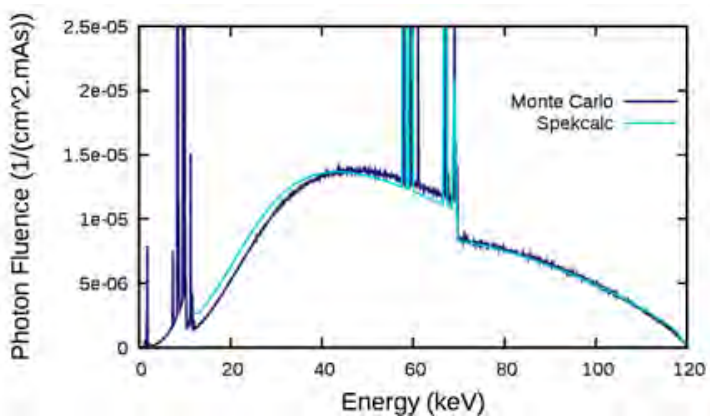

(a) Initial Spectra

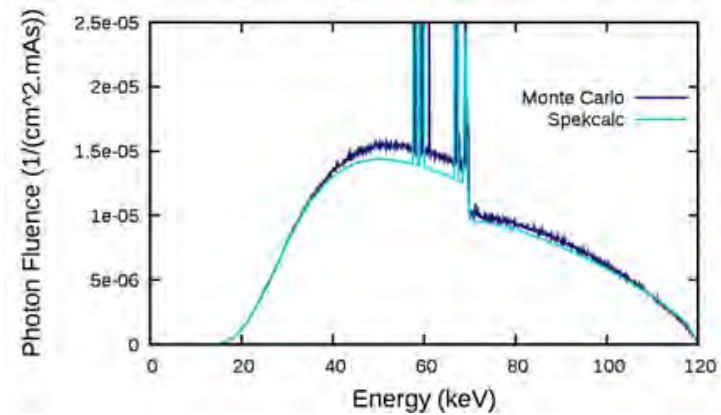

(b) Equivalent Spectra

Fig. 5. Initial and filtered spectra obtained with the iterative process.

$\mathrm{mm}$ in aluminium (MC spectrum) and set to $3.14410^{-5}$ for the equivalent filtration of $2.10 \mathrm{~mm}$ in aluminium (SpekCalc spectrum). Despite the good agreement on the inherent filtration and the two HVL, we note discrepancies of about $25 \%$ between the two simulated values (Table VI). The results obtained with the SpekCalc spectrum are closer to the measurements. Further investigations have to be performed to understand these discrepancies. One explanation could be the low interaction probability in air.

\begin{tabular}{ll}
\hline Measurements (mGy) & $574 \pm 15$ \\
Monte Carlo (mGy) & $714 \pm 29$ \\
SpekCalc (mGy) & $571 \pm 25$ \\
\hline
\end{tabular}

TABLE VI

RESULTS OBTAINED FOR THE AIR KERMA.

2) Equivalent Bowtie Filter: Body bowtie filters were designed to cover a large field of view (FOV) corresponding to adult abdominal CT. Head bowtie filters were designed to cover a small FOV corresponding to head CT or pediatric abdominal CT. We can note in Figure 6 that, according to theoretical expectation, the head bowtie filter (Figure 6(a)) appears narrower than the body bowtie filter (Figure 6(b)).

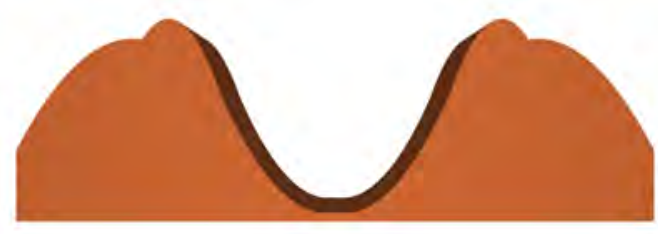

(a) Head bowtie filter

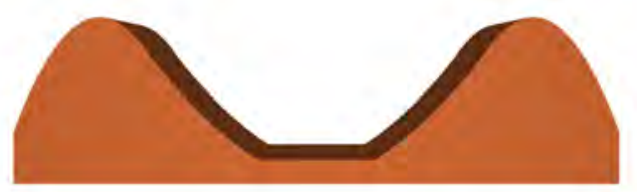

(b) Body bowtie filter

Fig. 6. PENGEOM 3D bowtie filter visualisation.

3) Geometric validation of the rotation: In Figure 7, multicolour points into the box represent photon emission point. Purple rectangle matches the ionization chamber used for measurements. As we can observe in Figure 7(a) points constitute a circle around the ionization chamber corresponding to an axial CT scan. Figure 7(b) shows a spiral rotation around the ionization chamber. Geometric validations for axial and spiral rotation look promising for further investigations in CT simulations.

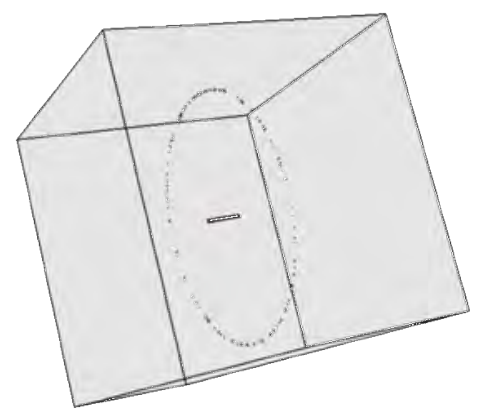

(a) Axial rotation

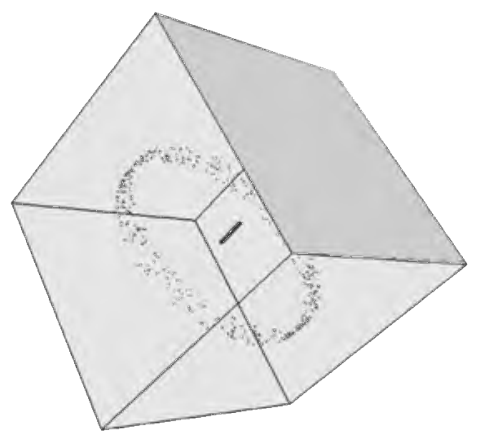

(b) Spiral rotation

Fig. 7. Geometric validation of CT rotation. 


\section{CONCLUSIONS}

The first results obtained with the PENRADIO software are encouraging. The validation of the program is part of the ongoing work for several phantoms and examination procedures in conventional and interventional radiography as well as in CT exams. We expect the same accuracy in the results that we have obtained up to now.

\section{APPENDIX}

\section{CALIBRATION AND CORRECTION OF OSL READINGS}

\section{A. OSL calibration}

There are two calibration curves according to the luminescent signal used at the time of reading (Figure 8): beam 1 for high doses and beam 2 for low doses. Readings corrected from the sensitivity of each OSL are used. Once this calibration is made the uncorrected air dose is obtained.

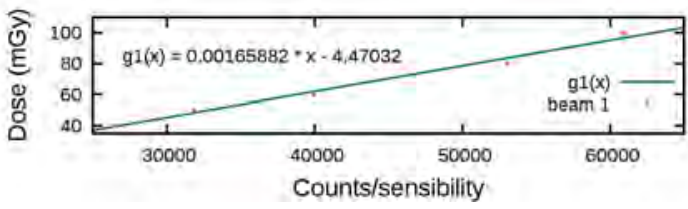

(a) Beam 1

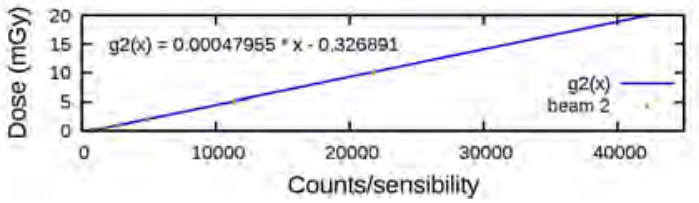

(b) Beam 2

Fig. 8. OSL calibrations.

\section{B. Energy response}

We determine the average energy of photons arriving on the OSL with a Monte Carlo simulation. The correction factor is determined using the curve (Figure 9) obtained in a preliminary study performed with a cobalt beam. We then obtain the corrected air dose.

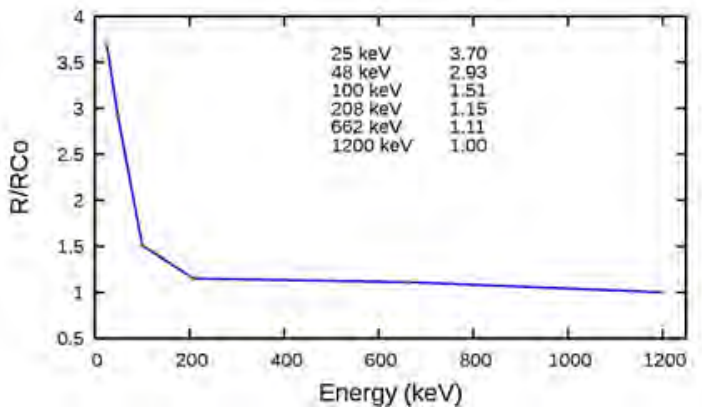

Fig. 9. Energy dependence of OSL detectors.

\section{ACKNOWLEDGMENTS}

The authors thank ESPRIMED for funding the work on dose calculation in conventionnal and interventionnal radiology.

\section{REFERENCES}

[1] P. C. Shrimpton, M. C. Hillier, M. A. Lewis, and M. Dunn, "Doses from computed tomography (ct) examinations in the uk - 2003 review," National Radiological Protection Board, 2005.

[2] R. Fazel, H. M. Krumholz, Y. Wanf, J. S. Ross, J. Chen, H. H. Ting, N. D. Shah, K. Nasir, A. J. Einstein, and B. K. NNallamothu, "Exposure to low-dose ionizing raditaion from medical imaging procedures," The New England Journal of Medicine, vol. 361, no. 9, pp. 849-857, 2009.

[3] C. Etard, S. Sinno-Tellier, and B. Aubert, "Exposition de la population franaise aux rayonnements ionisants lie aux actes de diagnostic mdical en 2007," 2010.

[4] J. Giles, "Study warns of 'avoidable' risks of ct scans," Nature, vol. 431, no. 7007, p. 391, 2004.

[5] D. J. Brenner and E. J. Hall, "Computed tomography - an increasing source of radiation exposure," The New England Journal of Medicine, vol. 357, no. 22, pp. 2277-2284, 2007.

[6] A. B. de Gonzalez, M. Mahesh, K. Kim, M. Bhargavan, R. Lewis, F. Mettler, and C. Land, "Projected cancer risks from computed tomographic scans performed in the united states in 2007," Arch Intern Med, vol. 169, no. 22, pp. 2071-2077, 2009.

[7] A. A. of Physicists in Medicine (AAPM), "The measurement, reporting, and management of radiation dose in ct, aapm report 96," 2008.

[8] http://www.stuk.fi.

[9] R. Kramer, "Caldose_x - a software tool for the assessmentn of organ and tissue absorbed doses, effective dose and cancer risks in diagnostic radiology," Phys Med Biol, vol. 53, pp. 6437-6459, 2008.

[10] "Eff-dose software, x-ray radiology dosimetry program using nrpb-sr262 national board of health, denmark, national institute of radiation hygiene," 1995

[11] E. K. Osei and R. Barnett, "Software for the estimation of organ equivalent and effective doses from diagnostic radiology procedures," $J$. Radiol. Prot, vol. 29, pp. 361-376, 2009.

[12] B. Schmitt and W. Kalender, "A fast voxel-based monte carlo method for scanner- and patient-specific dose calculations in computed tomography," Med. Phys, vol. 36, pp. 2898-2914, 2009.

[13] J. Jarry, J. J. DeMarco, C. H. Cagnon, and M. F. McNitt-Gray, "A monte carlo based method to estimate radiation dose from spiral ct: from phantom testing to patient-specific models," Phys. Med. Bio., vol. 48, pp. 2645-2663, 2009.

[14] J. J. DeMarco, C. H. Cagnon, D. D. Cody, D. M. Stevens, C. G. McCollough, J. O'Daniel, and M. F. McNitt-Gray, "A monte carlo based method to estimate radiation dose from multidetector ct : Cylindrical and anthropomorphic phantoms," Phys. Med. Bio., vol. 50, pp. 3989-4004, 2005.

[15] P. Deak, M. V. Straten, P. C. Shrimpton, M. Zankl, and W. A. Kalender, "Validation of a monte carlo tool for patient-specific dose simulations in multi-slice computed tomography," European Society of Radiology, vol. 18, pp. 759-772, 2008.

[16] X. Li, S. E., S. W. P., S. G.M., C. J.G., T. G., and F. D.P., "Patient-specific radiation dose and cancer risk estimation in ct: Part i. development and validation of a monte carlo program," Medical Physics, vol. 38, pp. 397-407, 2011.

[17] F. Salvat, J. F. Varea, and J. Sempau, PENELOPE 2006, A Code System for Monte Carlo Simulation of Electron and Photon Transport. OECD, (Barcelona, Spain), 2006.

[18] C. L. Loirec, J. C. G. Hernandez, G. Bonniaud, B. Poumarede, and D. Lazaro, "Dosimetric verification of penssart, a new monte carlo dose calculation system based on penelope and dedicated to patient-specific treatment quality control," MCTP2012 - May 15-18 2012 - Sevilla Spain, 2012.

[19] J. DeMarco, C. Cagnon, D. Cody, D. Stevens, C. McCollough, J. Daniel, and M. McNitt-Gray, "A monte carlo based method to estimate radiation dose from multidetector ct (mdct): cylindrical and anthropomorphic phantoms," Physics in Medicine and Biology, vol. 50, pp. 3989-4004, 2005.

[20] G. Bueno and O. Deniz, "Fast monte carlo simulation on a voxelized human phantom deformed to a patient," Medical Physics, vol. 36, pp. 5162-5174, 2009.

[21] P. Jursinic, "Characterization of optically stimulated luminescent dosimeters, oslds, for clinical dosimetric measurements," Medical Physics, vol. 34, pp. 4594-4604, 2007.

[22] ICRP, "The 1990 recommendations of the international commission on radiological protection," 1990.

[23] - "The 2007 recommendations of the international commission on radiological protection," 2007. 
[24] C. Turner, D. Zhan, H. Kim, and et al., "A method to generate equivalent energy spectra and filtration models based on measurement for multidetector ct monte carlo dosimetry simulations," Medical Physics, vol. 36, pp. 2154-2164, 2009.

[25] G. Poludniowski, "Calculation of x-ray spectra emerging from an x-ray tube. part ii. x-ray production and filtration in x-ray targets." Medical Physics, vol. 34, pp. 2175-2186, 2007. 